\title{
Vulnerabilidad de viviendas al viento de la localidad de ingeniero White, Argentina
}

\author{
Laura Veronica Gonzalez de paz ${ }^{1}$, Néstor Ortega, Marta Rosales \\ y Andres Salvatori
}

\section{Resumen}

Los mapas de riesgo para la identificación y evaluación de los efectos que algunos eventos naturales pueden ocasionar a la sociedad, son una herramienta indispensable en la gestión del riesgo y pueden ser utilizados fundamentalmente en el planeamiento urbano como así también en la respuesta ante estos sucesos ambientales naturales. Este trabajo es parte de un proyecto que tiene por objetivo de desarrollar metodologías para la generación de mapas de riesgo de construcciones residenciales e industriales menores, frente al viento, principalmente. El estudio se enfoca en la localidad de Ing. White, Argentina, una zona donde existe la posibilidad de que se presenten fenómenos extremos de viento, como corrientes descendentes y tornados. En esta etapa, se procedió a la caracterización de las construcciones a través de un relevamiento, considerando aspectos arquitectónicos y estructurales, estado de mantenimiento y posibles consecuencias de su falla. Los datos obtenidos se analizaron estadísticamente. Finalmente, y utilizando índices de vulnerabilidad físicos adecuados, existentes en la literatura, se elaboró un mapa de la vulnerabilidad de las viviendas.

Palabras clave: vulnerabilidad, viento, mapa de riesgo, construcciones.

1 Universidad Nacional del Sur/CONICET. 


\begin{abstract}
Risk maps to identify and assess the effects that certain type of natural events can cause to the society are an indispensable tool for the risk management and can be used mainly in urban planning as well as in the response to these natural environmental occurrences. This study is part of a larger project with the main aim of developing methodologies to generate wind risk maps of residential buildings and small industrial constructions. The study focuses at the location Ing. White, Argentina, in a region with probability of extreme wind events, as downbursts and tornados. At this stage, the constructions were classified, considering architectonic and structural aspects, preservation state and possible consequences of a failure. A statistic analysis of the data was performed. Finally, and using suitable vulnerability indices available in literature, a map with the geographic distribution of the vulnerability of the houses was obtained.
\end{abstract}

Keywords: vulnerability, wind, risk map, constructions. 


\section{Introducción}

Es conocida la importancia de planificar la distribución urbana y la respuesta frente a distintos eventos meteorológicos, algunos de los cuales pueden transformarse en desastres. Usualmente se orientan los esfuerzos a la atención de la emergencia y la reconstrucción. Sin embargo, debe propenderse a un sistema que, más allá de reaccionar frente al desastre, contemple la prevención. Para ello es necesario conocer los peligros (también llamados amenazas) y la vulnerabilidad de las construcciones, y así determinar el riesgo (Augusti et al, 2001; Stewart, 2003). Con este diagnóstico será posible diseñar políticas de planeamiento urbanístico a través del reconocimiento de zonas de asentamientos urbanos e infraestructura adecuadas, además de brindar la información apropiada a la población.

Frecuentemente existe una percepción de que el desastre es producto sólo de fenómenos naturales (ej. viento o sismo). Sin embargo, la sociedad se expone a dichos eventos a través de sus construcciones e infraestructura con distintos grados de vulnerabilidad, sumado a la proveniente de aspectos socioeconómicos. La combinación de los peligros y la vulnerabilidad tanto física como social, conforman el llamado riesgo (Nájera Herranz, 2005).

Se reconoce que un gran porcentaje de las pérdidas se deben a una inadecuada ubicación y diseño de las viviendas y de las obras de infraestructuras. Teniendo construcciones apropiadas, se puede reducir en un porcentaje importante el riesgo ante estos eventos naturales (Alcántara-Ayala, 2002; Khanduri \& Morrow, 2003). Típicamente, tres conceptos básicos se manejan y se combinan en esta temática (Lerner-Lam, 2007):

\section{Peligro (en inglés, Hazard) + Vulnerabilidad (Vulnerability $)=$ Riesgo (Risk)}

La modelación del daño, ante vientos extremos, se establece mediante funciones de vulnerabilidad, que tienen en cuenta la velocidad del viento pico, para ráfagas del orden de 5 segundos y la Relación Media de Daño que generan en las edificaciones. Para la evaluación de la vulnerabilidad de la edificación ante vientos muy fuertes se requiere información relativa al sistema estructural, elementos de fachada, tipo de cubierta, materiales, forma de la planta y altura de la construcción, influencia de edificios adyacentes, etc. Esta información, correlacionada con la vulnerabilidad social, dará una idea de la calidad de las construcciones y el daño que puede producir sobre los bienes afectados (Stewart, 2003; AAWE-ASCE, 2004; Unanwa et al, 2000).

En el Hemisferio Sur es muy poco frecuente que se generen huracanes y, en particular, en Argentina, es de interés el estudio de los tornados y las tormentas severas, que con frecuencia aparecen en distintos lugares del país (Reyes Salinas et 
al, 2009). En Bahía Blanca, en los años 1978 y 1982, dos tornados pasaron sobre la ciudad, afectando seriamente diversas construcciones que encontró en su recorrido por el área urbana. La zona de estudio pertenece a la llamada zona B, en la mapa de frecuencias de tornados reportado en (Schwarzkopf \& Rosso, 1993) También se han registrado otros fenómenos menos habituales como las corrientes descendentes o los "land-sprouts", que en Bahía Blanca en 2010, causaron múltiples daños en líneas de conducción eléctrica y en algunas edificaciones.

La velocidad del viento aumenta con la altura, sobre el nivel del terreno. La variación con la que se incrementa depende, no sólo de las condiciones de rugosidad del terreno, sino también de las ráfagas del viento. Las velocidades de las ráfagas suceden durante lapsos promedio del orden de 3 a 15 segundos y se relacionan con el tamaño de la estructura. Por ello, las construcciones esbeltas y flexibles se ven más afectadas por las ráfagas de corta duración, mientras que las bajas y rígidas son afectadas por las velocidades medias (Avelar Frausto, 2005; Eveleigh, 2007).

Los daños que típicamente presentan las construcciones afectadas por las altas velocidades del viento de los tornados, son (Stewart, 2003; Khanduri \& Morrow, 2003; Avelar Frausto, 2005):

- Caída de recubrimientos livianos en edificios.

- Rotura de ventanas.

- Daños a los grandes vitrales en la fachada de construcciones.

- Pérdida de techos livianos y daños en muros realizados en materiales livianos.

- Colapso de carteles comerciales, los cuales a su vez afectan construcciones cercanas.

- Rotura de muros, que por lo general están aislados.

- En ciertos casos, hasta la socavación de cimentaciones, en función del nivel de lluvias que acompañan a estos fenómenos eólicos.

Un índice de riesgo es un valor acotado entre cero y uno. Dicho índice sólo representa una medida cualitativa de la evaluación del riesgo. Es decir, es un indicador que detecta las zonas de una localidad que pueden tener mayor susceptibilidad al daño por la acción del viento. Si el índice se acerca a uno, significa que la construcción analizada es la que presenta la mayor susceptibilidad de daño. Este índice tiene dos componentes, el primero relacionado con la parte física y el segundo con los aspectos sociales (Avelar Frausto, 2005; Heneka \& Ruck, 2008).

Por otro lado, la manera formal de cuantificar la vulnerabilidad es a través de funciones de vulnerabilidad, que son una expresión matemática que relaciona las consecuencias probables de un fenómeno sobre una construcción, una obra de ingeniería, o un conjunto de bienes o sistemas, en función de la intensidad de dicho fenómeno (Heneka \& Ruck, 2008, - CENAPRED, 2006; ERN, 2009). 
De acuerdo con las posibles consecuencias de su falla, las construcciones se clasifican en (CENAPRED, 2006):

- Grupo A: construcciones cuya falla estructural podría causar la pérdida con un elevado costo económico, o en vidas o en bienes culturales (por ejemplo: hospitales, escuelas, museos); y

- Grupo B: construcciones ordinarias usadas como vivienda, oficinas y locales comerciales, hoteles y construcciones comerciales e industriales no incluidas en el Grupo A.

En este trabajo se reportan los avances realizados en un estudio sobre el riesgo frente al viento de las construcciones en una localidad de Argentina.

Como lo refleja el título del presente trabajo, se trata de hallar la vulnerabilidad al viento de viviendas en la localidad portuaria de Ingeniero White.

En la primera etapa se estudió toda la localidad, para lo cual se contó con la colaboración de estudiantes de Ingeniería Civil que realizaron un relevamiento in situ, con el fin de clasificar visualmente el tipo y estado de conservación de las construcciones. Para este relevamiento se desarrolló previamente una clasificación de viviendas, construcciones civiles e industriales y estructuras. De esta clasificación surgió una planilla que fue la utilizada en la encuesta realizada por los alumnos. Una vez completadas, se procedió a su procesamiento estadístico, cuyos resultados se muestran en gráficos que reflejan el perfil edilicio de la localidad estudiada.

Como herramienta de evaluación de los datos obtenidos, se utilizaron índices de vulnerabilidad cualitativos para viviendas, desarrollados en (CENAPRED, 2006). Si bien puede discutirse la pertinencia de la aplicación directa de estos índices, el objetivo fue utilizar la base de datos surgida del relevamiento para testear una posible zonificación. Respecto al peligro, dado el tamaño reducido de la región, se adoptó una única velocidad de viento, aunque sí se consideró una diferencia por efecto de la rugosidad en una zona del barrio donde existe una mayor exposición. El resultado de esta etapa es una base de datos de la localidad de Ingeniero White, que permite inferir el tipo y estado de las construcciones. Con estos datos y utilizando los índices arriba mencionados, se halló una distribución geográfica de la vulnerabilidad física en la localidad estudiada.

En un futuro se pretende desarrollar índices de vulnerabilidad a través de curvas de daño al viento para las tipologías más comunes. También se prevé un estudió localizado de las velocidades del viento a través de estaciones meteorológicas ya instaladas y otras a instalarse. 


\section{Motivación: daños por eventos meteorológicos en la zona previos al estudio}

Durante la realización de la primera parte del relevamiento, el 7 de diciembre de 2010, tuvo lugar en la ciudad de Bahía Blanca, y en particular en el barrio de Villa Rosas y en la localidad de Ing. White, una tormenta con vientos muy fuertes. De los datos de la estación meteorológica, se obtuvo que a las 6 p.m. de ese día (la estación reporta registros cada media hora) hubo una ráfaga máxima de 31,3 $\mathrm{m} / \mathrm{s}(113 \mathrm{~km} / \mathrm{h})$ en dirección WSW (OSO), con un valor de presión atmosférica que osciló entre 994-998 bars, en la hora previa. La temperatura era de $27,2^{\circ} \mathrm{C}$ y la humedad del $54 \square$. En la Figura 1 se muestra la imagen satelital infrarroja de la tormenta sobre el sur de la Pcia. de Buenos Aires donde se encuentra la ciudad de Bahía Blanca y, en particular, la zona de estudio (Ing. White).

La entidad de Bomberos Voluntarios de Ing. White realiza un registro de todas las salidas de su personal ante las demandas de la población. Del parte de servicios del día del evento en cuestión y de la zona en estudio y sus alrededores, se extrajo el siguiente detalle: 25 árboles caídos (algunos sobre autos y viviendas), 9 voladuras de techos, 5 caídas de cables, 9 caídas de postes, 3 caídas de carteles publicitarios, entre otros accidentes. En particular, hubo daño en una estructura de galpón de dimensiones importantes con dos heridos y otro herido por caída de un paredón en la línea municipal. En la Figura 2 se muestran algunas fotografías correspondientes a esos eventos. Debe notarse que este evento no es una situación aislada ya que este tipo de tormentas suele repetirse y en los últimos años han sucedido, en promedio, más de dos eventos de este tipo en el año, en Bahía Blanca y su zona de influencia.

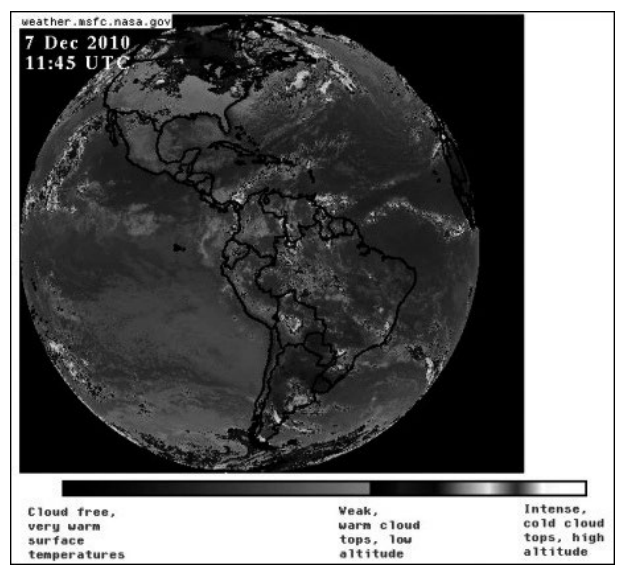

Figura 1. Imagen satelital infrarroja GOES-E correspondiente al día 7 de diciembre de 2010, sobre la República Argentina (Satélite GOES). 
Se evidencia que esta zona geográfica se encuentra expuesta y es vulnerable a este tipo de amenazas meteorológicas, resultando en daños que afectan operativamente y económicamente a la población y en algunos casos, representan peligro para la integridad de sus habitantes, como lo es la caída de cables y paredones, voladura de techos, etc. Además de la movilización de los entes de respuesta ante las emergencias (Bomberos, Policía, Defensa Civil), el conocimiento preventivo de la vulnerabilidad y su distribución geográfica, permitiría una mejor organización y calidad de la respuesta.

\section{Clasificación de viviendas y construcciones}

Mediante una visita preliminar, se registraron las tipologías más frecuentes a través de fotografías y en base a ello, se desarrolló una clasificación que comprendió tres grandes grupos: viviendas familiares, construcciones públicas y estructuras. A su vez, cada grupo fue sub-clasificado. Se plasmó dicha clasificación en sendas planillas cuyo contenido pueden verse en la Tabla 1. Alumnos de Ingeniería Civil, bajo la supervisión de los autores de este trabajo, realizaron un relevamiento de la localidad de Ingeniero White. Esta localidad se encuentra cercana a la costa marina sobre el Estuario de Bahía Blanca, ubicado en el extremo norte de la Patagonia Argentina. Se trata de un emplazamiento urbano, próximo a una zona portuaria, donde también se ubica el Polo Petroquímico más importante de Argentina, así como uno de sus principales puertos de ultramar. Por las actividades que se desarrollan en las proximidades, puede apreciarse la existencia de una zona intermedia destinado a maniobras ferroviarias y depósitos de materiales, en su mayoría a granel, para importación y exportación.

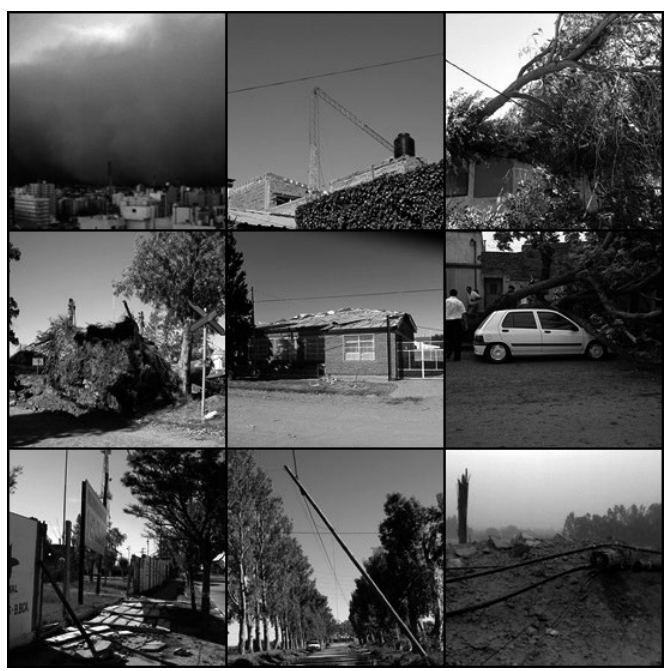

Figura 2. Imágenes del evento del 7 de diciembre de 2010 sobre Bahía Blanca e Ing. White y sus consecuencias. Fuentes propias y Diario "La Nueva Provincia" (edición digital). 
En la Figura 3 se muestra un plano de la ubicación de la localidad en estudio, y su ubicación dentro del territorio argentino. En el relevamiento, los alumnos utilizaron la planilla de la Tabla 2 y recorrieron todas las manzanas clasificando las construcciones. De las 422 ha que tiene la zona en total, se contabilizaron 214 ha edificadas, distribuidas en 160 manzanas.

Tabla 1. Tipologías de estructuras relevadas
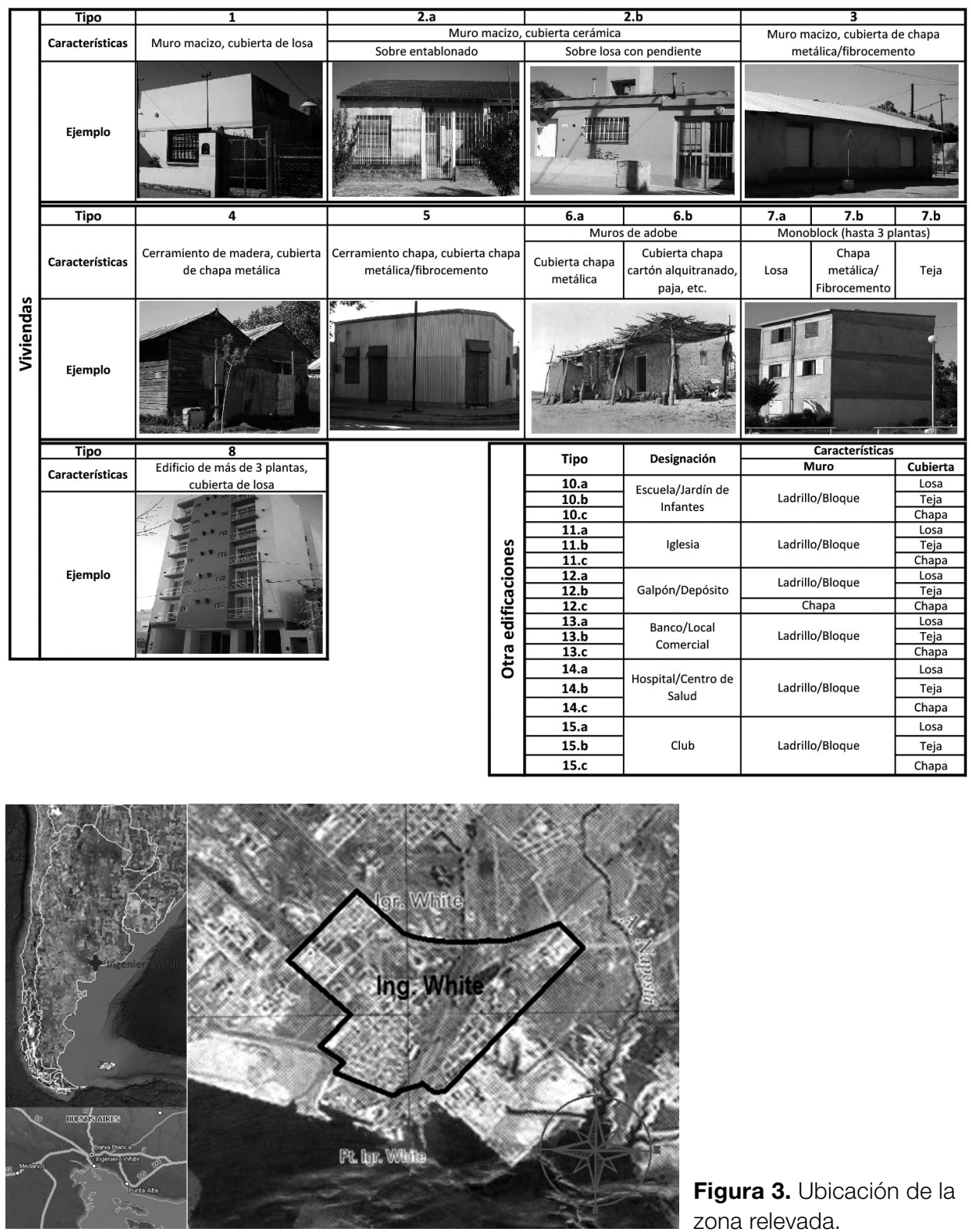

Figura 3. Ubicación de la zona relevada. 
Tabla 2. Planillas usadas en el relevamiento.
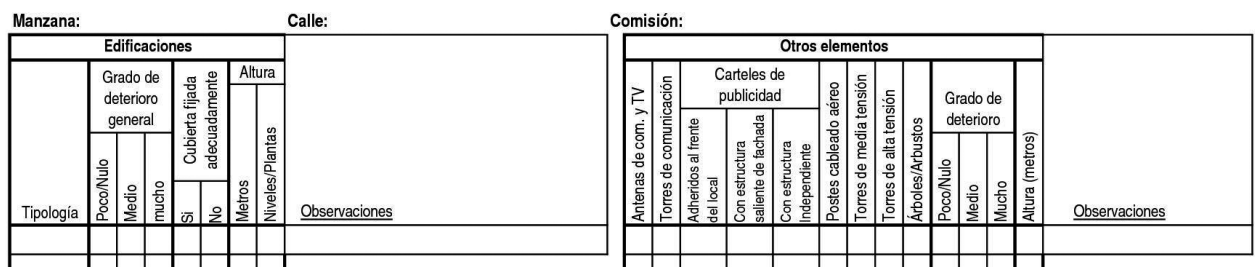

Los resultados volcados en las planillas fueron luego analizados, complementando estos datos con imágenes satelitales provenientes de la herramienta GOOGLE EARTH. De ellos se infirieron gráficos, con los que se realizaron los distintos análisis estadísticos que permiten estudiar el perfil de las construcciones (Figura 4).

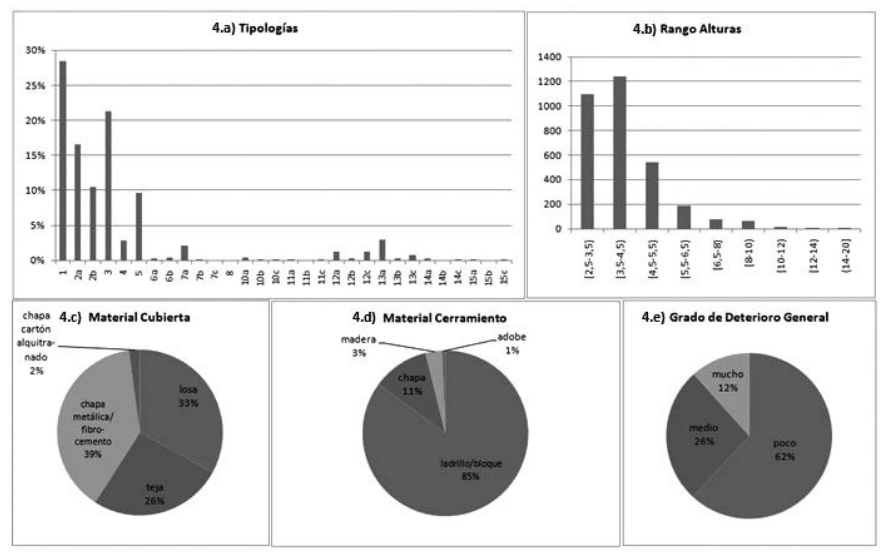

Figura 4. Gráficos de datos relevados.

La Figura 4.a muestra la frecuencia de las tipologías de edificación relevadas, observándose un predominio de viviendas (tipologías 1 a 8 , cf. Tabla 1). La Figura 4.b contiene la distribución de alturas de las viviendas, deduciéndose un perfil de viviendas de una y dos plantas como predominantes, es decir, viviendas unifamiliares. En la Figuras 4.c y 4.d pueden observarse los materiales usados en muros de cerramiento y para cubierta. Puede verse que mientras que la mayoría de las construcciones ofrecen mayor seguridad frente a vientos de magnitud importante por los materiales usados para cerramientos (predominantemente, ladrillo o bloque), no sucede así con los materiales usados en cubiertas (chapas y tejas, se infieren como más vulnerables). La Figura 4.e muestra el estado de deterioro general de las edificaciones, notándose que, aunque predominan las edificaciones poco deterioradas, existe un porcentaje no despreciable de edificaciones con gran deterioro. 


\section{Evaluación de los índices de vulnerabilidad}

A los efectos de verificar los datos obtenidos, se utilizan los índices cualitativos de vulnerabilidad física de viviendas presentados en (CENAPRED, 2006). Si bien estos índices fueron desarrollados para tipologías de México, se adoptaron sus valores en esta etapa, al no contarse con índice propios de la zona y dada la similitud de las viviendas consideradas en ese estudio, con las relevadas en la localidad de Ingeniero White. Para asignar los valores de los índices se adoptaron, en primera instancia, aquellos calculados en (CENAPRED, 2006) para los elementos de muros de cerramiento y cubierta en forma separada. El valor correspondiente a cada tipología se calculó según la expresión:

$$
I V F_{\text {edif }}=\frac{I V F_{\text {cub }}+I V F_{\text {muro }}}{2}
$$

donde $I V F_{\text {edif }}$ es el Índice de Vulnerabilidad Física de la edificación, $I V F_{c u b}$ es el Índice de Vulnerabilidad Física de la cubierta e $I V F_{\text {muro }}$ es el Índice de Vulnerabilidad Física de los muros de cerramiento. Para más de un nivel se adiciona el valor de muro con cubierta flexible si el muro es flexible y con cubierta rígida si el muro es rígido, tantas veces como niveles adicionales se consideren. La fórmula anterior pasa a ser:

$$
I V F_{\text {edif }}=\frac{I V F_{\text {cub }}+I V F_{\text {murosup }}+I V F_{\text {muroinf }} \times \text { nro pisos }}{2+\text { nro pisos }}
$$

Como velocidad regional se adoptó la velocidad básica para Bahía Blanca indicada por el CIRSOC 102 (INTI CIRSOC, 2005) $(\mathrm{V}=55 \mathrm{~m} / \mathrm{s}=200 \mathrm{~km} / \mathrm{h}$ ). Para considerar la rugosidad en forma global, debido a que es una variable que intervino en los cálculos, se dividió el área relevada en dos sectores: a) área urbana con densidad apreciable de edificaciones; b) campo abierto, con poca densidad de edificaciones. Los valores indican como 0 (cero) a vulnerabilidad mínima y a 1 (uno) como vulnerabilidad máxima (Tabla 3). Para las cubiertas de losa se considera una vulnerabilidad nula. Para cubierta flexible (teja sobre entablonado y chapa) se tomó un mismo valor, independiente del material, con una separación entre clavaderas de 60 a $70 \mathrm{~cm}$, usual en las construcciones locales. Para cubierta de tejas sobre losa inclinada se tomo un $30 \%$ del valor adoptado para cubierta flexible, teniendo en cuenta la posibilidad de desprendimiento de elementos, pero con muy poca probabilidad de voladuras de techos. Para los cerramientos de chapa o madera se tomaron los valores correspondientes a elementos prefabricados. Este tipo de vivienda con paredes de chapa o madera es típica en las localidades portuarias en Sudamérica. No se consideró en la determinación de los valores, el grado de deterioro de las edificaciones. 
Una vez obtenidos los valores para cada tipología (Tabla 4), el siguiente paso fue determinar un valor de vulnerabilidad por manzana $\mathrm{IV}_{\text {manzana }}$ que se calculó como la media de los valores de las edificaciones en dicha unidad de suelo, llamándose $\mathrm{N}$ al número de edificaciones existentes por manzana:

$$
I V_{\text {manzana }}=\frac{\sum_{i=1}^{N} I V F_{\text {edif }}}{N}
$$

También se adoptó una escala de vulnerabilidades de acuerdo a los valores de los índices resultantes, según se detalla en la Tabla 3. De la aplicación de estos niveles a los datos del relevamiento, se obtuvo la distribución geográfica de los índices de vulnerabilidad que se reflejó en el mapa de la Figura 5, en un esquema de colores. Debe notarse que las manzanas que aparecen sin colorear, corresponden a zonas sin construcciones.

Tabla 3: Escala de Vulnerabilidad (CENAPRED, 2006).

\begin{tabular}{|l|l|}
\hline Valor $\mathrm{IV}_{\text {mza }}$ & Nivel de riesgo \\
\hline $0,0 \leq \mathrm{IV}<0,2$ & Muy bajo \\
\hline $0,2 \leq \mathrm{IV}<0,4$ & Bajo \\
\hline $0,4 \leq \mathrm{IV}<0,6$ & Medio \\
\hline $0,6 \leq \mathrm{IV}<0,8$ & Alto \\
\hline $0,8 \leq \mathrm{IV}<1,0$ & Muy alto \\
\hline
\end{tabular}

\section{Resultados obtenidos y comentarios finales}

La Tabla 4 expone los valores resultantes de los Índices de Vulnerabilidad Física para las edificaciones clasificadas (cf. Tabla 1), luego de aplicar las condiciones propuestas anteriormente, y para una velocidad básica de $200 \mathrm{~km} / \mathrm{h}$. En la Figura 5 se observa el Mapa de Vulnerabilidad resultante del estudio de los datos relevados, para dicha velocidad. También se reportan mapas correspondientes a velocidades por encima de la de referencia $(220 \mathrm{~km} / \mathrm{h}, 240 \mathrm{~km} / \mathrm{h}, 260 \mathrm{~km} / \mathrm{h}$ y $280 \mathrm{~km} / \mathrm{h})$. Cabe acotar que en determinadas corrientes descendentes, de acción relativamente localizada, se llegan a generar vientos de hasta $270 \mathrm{~km} / \mathrm{h}$ (Schwarzkopf \& Rosso, 1993). 
Tabla 4: Índices de Vulnerabilidad para edificaciones relevadas para una velocidad básica de $200 \mathrm{~km} / \mathrm{h}$.

\begin{tabular}{|c|c|c|c|c|c|c|}
\hline \multirow{3}{*}{ Tipología } & \multicolumn{2}{|c|}{ Cubierta } & \multicolumn{2}{|c|}{ Muro de cerramiento } & \multirow{2}{*}{\multicolumn{2}{|c|}{$\frac{\text { Muro (para más de } 1 \text { nivel) }}{\text { Sector }}$}} \\
\hline & \multicolumn{4}{|c|}{ Sector } & & \\
\hline & Urbano & Abierto & Urbano & Abierto & Urbano & Abierto \\
\hline 1 & 0,0000 & 0,0000 & 0,0234 & 0,0857 & 0,0234 & 0,0857 \\
\hline $2 a$ & 0,0987 & 0,3596 & 0,2259 & 0,7650 & 0,0234 & 0,0857 \\
\hline $2 b$ & 0,0296 & 0,1079 & 0,0234 & 0,0857 & 0,0234 & 0,0857 \\
\hline $3 a$ & 0,0987 & 0,3596 & 0,2259 & 0,7650 & 0,0234 & 0,0857 \\
\hline $3 b$ & 0,0987 & 0,3596 & 0,2259 & 0,7650 & 0,0234 & 0,0857 \\
\hline 4 & 0,0987 & 0,3596 & 0,0083 & 0,9993 & 0,0234 & 0,0857 \\
\hline $5 a$ & 0,0987 & 0,3596 & 0,0083 & 0,9993 & 0,0083 & 0,9993 \\
\hline $5 b$ & 0,0987 & 0,3596 & 0,0083 & 0,9993 & 0,0083 & 0,9993 \\
\hline $6 a$ & 0,0987 & 0,3596 & 0,0008 & 0,0229 & 0,0083 & 0,9993 \\
\hline $6 b$ & 0,0987 & 0,3596 & 0,0008 & 0,0229 & 0,0083 & 0,9993 \\
\hline $7 a$ & 0,0000 & 0,0000 & 0,0234 & 0,0857 & 0,0234 & 0,0857 \\
\hline $7 \mathrm{~b}$ & 0,0987 & 0,3596 & 0,2259 & 0,7650 & 0,0234 & 0,0857 \\
\hline $7 c$ & 0,0987 & 0,3596 & 0,2259 & 0,7650 & 0,0234 & 0,0857 \\
\hline 8 & 0,0000 & 0,0000 & 0,0234 & 0,0857 & 0,0234 & 0,0857 \\
\hline $10 a$ & 0,0000 & 0,0000 & 0,0234 & 0,0857 & 0,0234 & 0,0857 \\
\hline $10 b$ & 0,0987 & 0,3596 & 0,2259 & 0,7650 & 0,0234 & 0,0857 \\
\hline $10 c$ & 0,0987 & 0,3596 & 0,2259 & 0,7650 & 0,0234 & 0,0857 \\
\hline $11 a$ & 0,0000 & 0,0000 & 0,0234 & 0,0857 & 0,0234 & 0,0857 \\
\hline $11 b$ & 0,0987 & 0,3596 & 0,2259 & 0,7650 & 0,0234 & 0,0857 \\
\hline $11 c$ & 0,0987 & 0,3596 & 0,2259 & 0,7650 & 0,0234 & 0,0857 \\
\hline $12 a$ & 0,0000 & 0,0000 & 0,0234 & 0,0857 & 0,0234 & 0,0857 \\
\hline $12 b$ & 0,0987 & 0,3596 & 0,2259 & 0,7650 & 0,0234 & 0,0857 \\
\hline $12 c$ & 0,0987 & 0,3596 & 0,2259 & 0,7650 & 0,0234 & 0,0857 \\
\hline $12 d$ & 0,0987 & 0,3596 & 0,0083 & 0,9993 & 0,0083 & 0,9993 \\
\hline $12 e$ & 0,0987 & 0,3596 & 0,0083 & 0,9993 & 0,0083 & 0,9993 \\
\hline $13 a$ & 0,0000 & 0,0000 & 0,0234 & 0,0857 & 0,0234 & 0,0857 \\
\hline $13 b$ & 0,0987 & 0,3596 & 0,2259 & 0,7650 & 0,0234 & 0,0857 \\
\hline $13 c$ & 0,0987 & 0,3596 & 0,2259 & 0,7650 & 0,0234 & 0,0857 \\
\hline $14 a$ & 0,0000 & 0,0000 & 0,0234 & 0,0857 & 0,0234 & 0,0857 \\
\hline $14 b$ & 0,0987 & 0,3596 & 0,2259 & 0,7650 & 0,0234 & 0,0857 \\
\hline $14 c$ & 0,0987 & 0,3596 & 0,2259 & 0,7650 & 0,0234 & 0,0857 \\
\hline $15 a$ & 0,0000 & 0,0000 & 0,0234 & 0,0857 & 0,0234 & 0,0857 \\
\hline $15 b$ & 0,0987 & 0,3596 & 0,2259 & 0,7650 & 0,0234 & 0,0857 \\
\hline $15 c$ & 0,0987 & 0,3596 & 0,2259 & 0,7650 & 0,0234 & 0,0857 \\
\hline
\end{tabular}




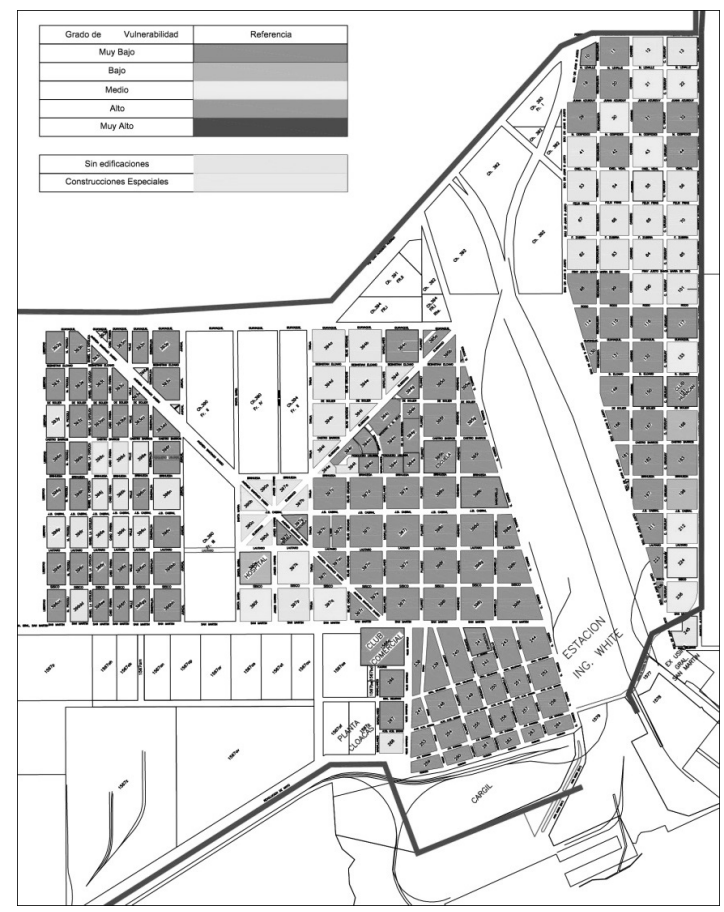

Figura 5. Mapa de vulnerabilidad de zona relevada para velocidad básica de $200 \mathrm{~km} / \mathrm{h}$.

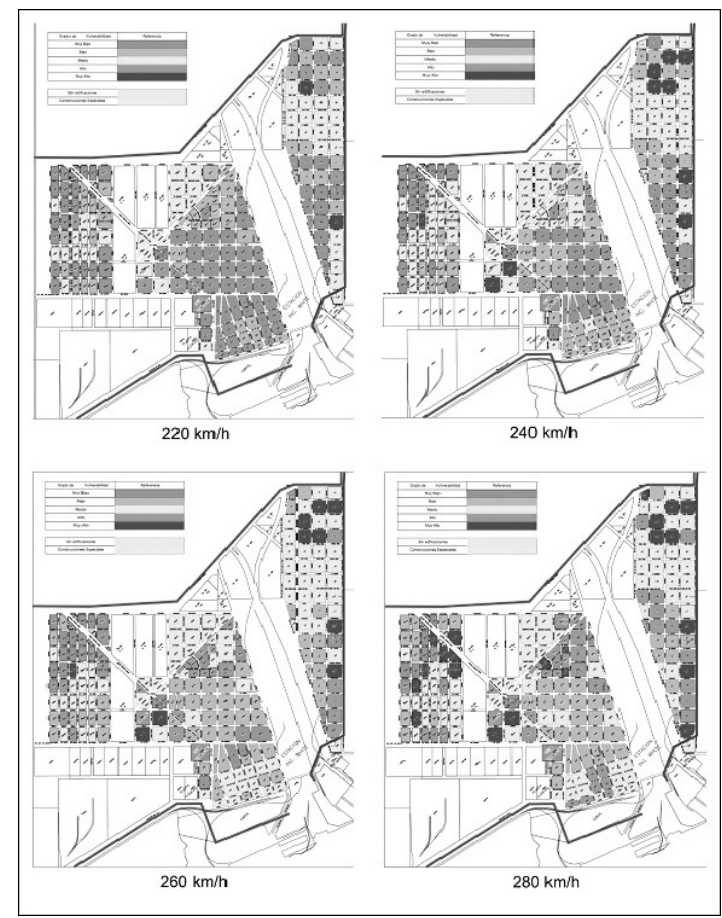

Figura 6. Mapa de vulnerabilidad de zona relevada para velocidades mayores de $200 \mathrm{~km} / \mathrm{h}$. 
En estos mapas puede advertirse que el nivel de vulnerabilidad, en general, es bajo y sólo en algunos sectores, más sensibles al viento, aparece un mayor nivel. Justamente, estos sectores coinciden con las de mayor vulnerabilidad social. Por ello, desgraciadamente, cuando hay catástrofes naturales, las regiones o los países más pobres son los más damnificados, tal que su recuperación suele ser más difícil, no solo por tener una menor capacidad económica, sino que también los daños que se producen en sus construcciones son mayores que si el mismo evento, hubiese afectado a lugares con más poder económico.

En este trabajo, se reportan los resultados de estudios preliminares realizados en una localidad portuaria de Argentina, referido al análisis del riesgo de construcciones frente a eventos eólicos extremos. El estudio incluyó una clasificación de construcciones, la elaboración de planillas para relevamiento y los resultados volcados en gráficos estadísticos. Además, se utilizaron índices de vulnerabilidad ya publicados por otros autores para situaciones similares. Se logró obtener un plano de zonificación de la vulnerabilidad física. El estudio continúa con la elaboración de índices específicos de las construcciones del lugar.

Este tipo de estudios es una importante herramienta en la planificación urbana y en la planificación para la respuesta ante eventos eólicos extremos, que también se puede aplicar en otras ciudades de América Latina y realizando adaptaciones, en todo lo referente a las tipologías de construcciones existentes, también sería aplicable a ciudades de países desarrollados, expuestos a estas acciones naturales. 


\section{Bibliografía}

Augusti, G., Borri, C., Niemann, H.J.,(2001). Is Aeolian risk as significant as other environmental risks?. Reliability Engineering System Safety, Vol. 74, pp. 227-237.

Stewart, M.G., (2003). Cyclone damage and temporal changes to building vulnerability and economic risks for residential construction. Journal of Wind Engineering and Industrial Aerodynamics, Vol. 91 no 5, pp. 671-691.

Nájera Herranz, J.C. (2005). El mapa de riesgos de la naturaleza. MAPFRE. Gerencia de riesgos y seguros. División de Grandes Riesgos, Vol. 23 no 91, pp. 15-30.

Alcántara-Ayala, I. (2002). Geomorphology, natural hazards, vulnerability and prevention of natural disasters in developing countries. Geomorphology, Vol. 47, pp. 107-124.

Khanduri, A.C. and Morrow, G.C., (2003). Vulnerability of buildings to windstorms and insurance loss estimation. Journal of Wind Engineering and Industrial Aerodynamics, Vol. 91 no 4, pp. 455-467.

Lerner-Lam, A. (2007). Assessing global exposure to natural hazards: Progress and future trends. Environmental Hazards, Vol. 7 no 1, pp. 10-19.

AAWE-ASCE , (2004). Challenge of growing vulnerability to wind hazards in the US and opportunities to reverse this trend (abbreviated report). The Wind Engineer. American Association for Wind Engineering.

Unanwa, C.O. and McDonald, J.R. and Mehta, K.C. and Smith, D.A., (2000). The development of wind damage bands for buildings. Journal of Wind Engineering and Industrial Aerodynamics, Vol. 84, pp. 119-149.

Reyes Salinas C., Flores Corona L., Pacheco Martínez M.A., López Bátiz O., Valerio Zárate L.A. y Zepeda Ramos O. (2009). Evaluación simplificada de la vulnerabilidad de la vivienda unifamiliar ante sismo y viento. Universidad de Guadalajara, México.

Schwarzkopf M.L. y Rosso L.C. (1993). Riesgo de Tornados y Corrientes Descendentes en la Argentina", INTI CIRSOC, Buenos Aires.

Avelar Frausto C. (2005). Estimación de pérdidas en México por riesgos hidrometeorológicos. Vulnerabilidad de estructuras ante viento. ERN, México.

Eveleigh, T.J. and Mazzuchi, T.A. and Sarkani, S. (2007). Spatially-aware 
systems engineering design modeling applied to natural hazard vulnerability assessment. Systems Engineering, Vol. 10 no 3, pp.187-202.

Heneka, P. and Ruck, B.A. (2008). Damage model for the assessment of storm damage to buildings. Engineering Structures, Vol. 30 no 12, pp. 3603-3609.

Centro Nacional de Prevención de Desastres CENAPRED (2006). Guía básica para la elaboración de atlas estatales y municipalidades de peligros y riesgos. Fenómenos meteorológicos", México.

Consorcio Evaluación de Riesgos Naturales-América Latina ERN, CAPRA. (2009). Nicaragua .Tomo I. Metodología de Modelación Probabilística de Riesgos Naturales. Vulnerabilidad de Edificaciones e Infraestructura. Informe Técnico ERN-CAPRA-T1-5.

Satelite GOES. http://wwwghcc.msfc.nasa.gov, acceso 08/12/2010.

Centro de Investigación de los Reglamentos Nacionales de Seguridad para las Obras Civiles, (2005). CIRSOC 102 - Reglamento Argentina de Acción del Viento sobre las Construcciones, INTI CIRSOC, Buenos Aires. 